\title{
Educação Tutorial em Computação: Uma Análise Retrospectiva das Atividades Realizadas para a Formação Pessoal e Profissional de Cientistas da Computação
}

\author{
Fernando Leite', Gabriel Cardoso ${ }^{1}$, Yandson Costa ${ }^{1}$, Igor Estrela ${ }^{1}$, Antônio Pinto ${ }^{1}$, \\ Ednara Pereira ${ }^{1}$, Geraldo Braz Júnior ${ }^{2}$, Luis Rivero ${ }^{1,2}$ \\ ${ }^{1}$ Programa de Educação Tutorial de Ciência da Computação (PETComp) \\ Universidade Federal do Maranhão (UFMA) \\ ${ }^{2}$ Núcleo de Computação Aplicada (NCA) \\ Universidade Federal do Maranhão (UFMA) \\ \{fernandojo,gabri.elmc15031999, yandson.jesus,igor.star.ie, antoniomorar \\ a, ednara.asp\}@gmail.com, \{geraldo, luisrivero\}@nca.ufma.br
}

Abstract. The Programa de Educação Tutorial (PET) acts as an integrated educational initiative which intends to suppress evasion, encourage creative solutions and entrepreneurship, as well as complement new degrees of academic knowledge. Even though there are 37 PET groups according to the Sociedade Brasileira de Computação (SBC), few groups currently publish their activities' results through computer science conferences as a way to discuss their results and implications. This paper presents an analysis of the activities of a PET Ciencia da Computação group from the students' point of view. The results of the activities show their impact through the students' education, lessons learnt and improvement opportunities.

Resumo. O Programa de Educação Tutorial (PET) atua como uma iniciativa de promover a formação integrada de todos os atores envolvidos combatendo a evasão, estimulando o desenvolvimento criativo e empreendedor, assim como a continuação para níveis superiores de formação acadêmica. Apesar da existência de 37 grupos PET na área de computação segundo a Sociedade Brasileira de Computação (SBC), poucos grupos divulgam os resultados das ações realizadas em eventos científicos da computação para discutir suas implicações. Nesse contexto, este trabalho apresenta uma análise das atividades desenvolvidas em um grupo PET de Ciência da Computação do ponto de vista dos discentes. Os resultados apontam o impacto das atividades na formação dos alunos, lições aprendidas e oportunidades de melhoria para o sucesso das atividades.

\section{Introduçãa}

Segundo o relatório do censo da educação superior do Ministério da Educação ${ }^{1}$, alunos iniciantes em um curso de graduação enfrentam uma grande dificuldade em se manter em um curso devido ao nível acadêmico exigido e à falta de acompanhamento. Visando diminuir estas dificuldades, o Programa de Educação Tutorial (PET) foi criado para aprimorar os cursos de graduação, inserindo estudantes em projetos de educação tutorial,

\footnotetext{
${ }^{1}$ http://www.donaknow.com.br
} 
com o objetivo de aplicar seus conhecimentos e sua formação [Martins 2007]. O PET trabalha com três vertentes principais, que são: Ensino, Pesquisa e Extensão; formando cidadãos que aprendem a trabalhar em equipe, transmitindo aos demais colegas o espírito de liderança e o compromisso com a geração de conhecimento para a solução dos mais diversos problemas. Segundo o Ministério da Educação (MEC), o PET trabalha no formato de grupo interdisciplinar, ancorado a alunos e professores, que recebe avaliação institucional.

Os grupos PET têm se mostrado muito eficaz para diminuir os altos índices de evasão dos discentes em cursos de graduação [Deus Júnior et al. 2014]. As pesquisas que são desenvolvidas pelos seus integrantes têm por intuito levar o conhecimento das áreas de interesse dos mesmos à comunidade, agregando neste processo o saber e experiências adquiridas através de cursos e projetos de extensão. As ações levadas à comunidade são arreigadas de conhecimento e colhem como fruto o interesse dos jovens que entrarão no universo acadêmico, sendo assim estes já terão consigo consciência de como será o seu desenvolvimento ao atuar de forma ativa, tornando, portanto, sua evasão reduzida.

Dentre os grupos nas áreas de exatas, especificamente informática, existem 37 grupos PET que desenvolvem diversas atividades [Referência: portal da SBC]. Aplicando a tríade do conhecimento (ensino, pesquisa e extensão), o grupo PET Ciência da Computação da Universidade Federal do Maranhão (UFMA) tem um papel fundamental na formação dos seus integrantes, colocando-os em contato com todos os campos de atuação que o curso de Ciência da Computação pode proporcionar. Assim, o grupo tem se mostrado uma ponte entre o curso e os laboratórios de pesquisa da universidade, dispondo atividades que proporcionam imersão no conhecimento acadêmico, assim como vivência e experiência que são requisitadas no mercado de trabalho. Nesse contexto, este artigo apresenta um relato de experiência das principais ações realizadas pelo grupo PET Ciência da Computação, indicando os resultados obtidos, uma análise de acordo com ponto de vista dos discentes pertencentes ao grupo e outros beneficiados com as ações realizadas. Assim, espera-se expor as experiências positivas e negativas do contexto das atividades, servindo como referência a trabalhos futuros.

O restante deste artigo está organizado como segue. A Seção 2 apresenta as ações realizadas no grupo PET Ciência da Computação, apresentando suas motivações e metodologias. A Seção 3 apresenta uma discussão dos resultados alcançados pelas ações realizadas comparando-as com ações/iniciativas reportadas por outros grupos PET. Finalmente, a Seção 4 apresenta as conclusões e perspectivas futuras para este artigo.

\section{Ações}

Esta seção apresenta as principais intervenções desenvolvidas pelo PET Ciência da Computação da UFMA, que promovem e fortalecem o ensino da computação e o desenvolvimento pessoal e profissional dos discentes.

\subsection{Fábrica de Software}

Embora os cursos de computação ofereçam disciplinas que permitam ao aluno se inserir no mercado de trabalho conhecendo metodologias de desenvolvimento de software e suas aplicações, ainda é necessário incluir conteúdos a respeito de tecnologias atuais [Moreno et al., 2012]. Nesse contexto, criou-se o projeto Fábrica de Software, visando que os alunos do curso pudessem aplicar, na prática, conceitos de Engenharia de Software, 
Interação Humano-Computador, Algoritmos, Estruturas de Dados e Programação Avançada de Sistemas. O objetivo é que os alunos possam desenvolver sistemas computacionais para diversos contextos, sejam administrativos, entretenimento, pesquisa, entre outros.

Nesta atividade são mantidos pequenos grupos de desenvolvimento de software, que atuam na coleta, criação e manutenção de softwares inovadores, sejam eles para a universidade ou para o mercado. Assim, entende-se que a primeira área de atuação da atividade é a construção de objetos tecnológicos. Além disso, a atividade visa a continuação e posterior integração dos petianos em laboratórios de pesquisa, para dar continuidade à carreira acadêmica.

As ferramentas que foram e estão sendo desenvolvidas pelo projeto são amplamente disponibilizadas e registradas de forma aberta para que outras instituições, caso tenham interesse, possam utilizá-las. As Figuras 1 e 2 apresentam telas de dois dos sistemas que foram desenvolvidos como resultado desta atividade: (1) um sistema de controle financeiro interno do PET Ciência da Computação e (2) um sistema de gerenciamento de eventos realizados dentro e fora da universidade.

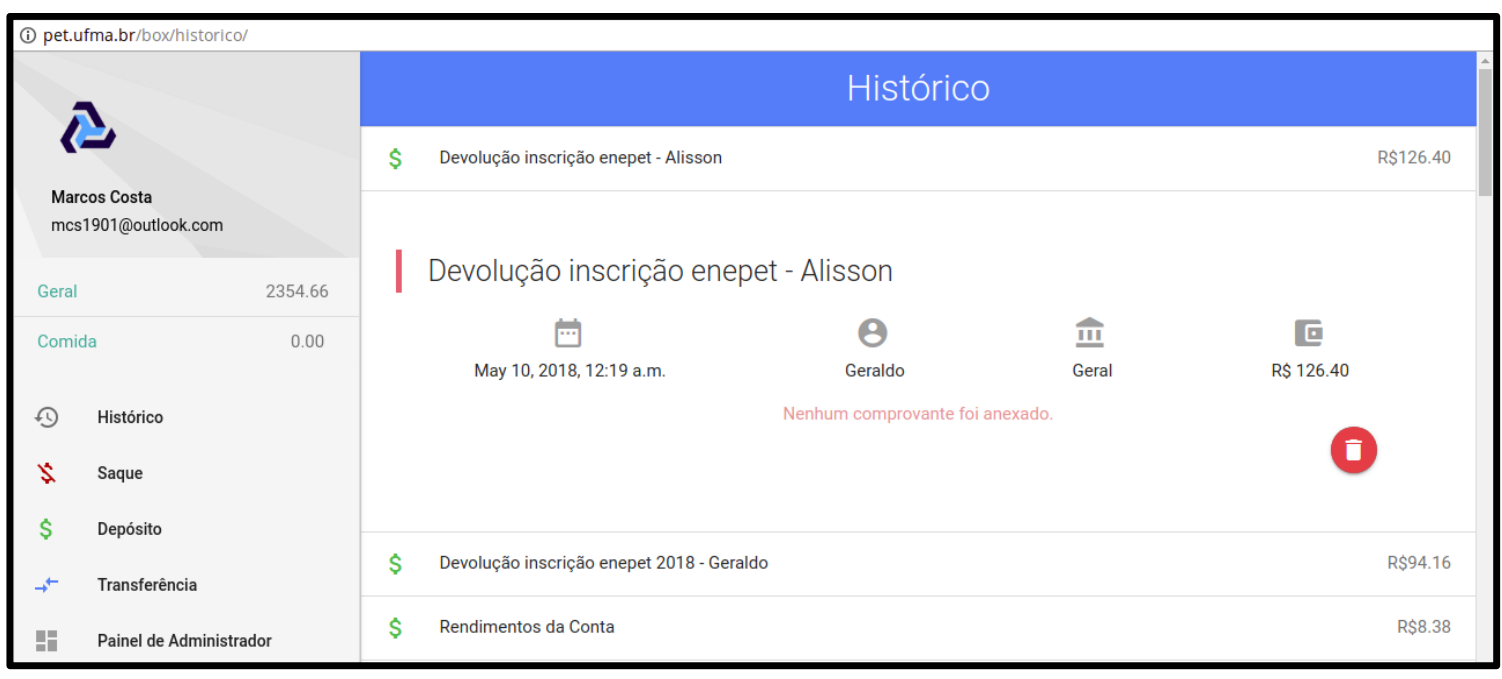

Figura 1. Safebox - Sistema desenvolvido para controle financeiro

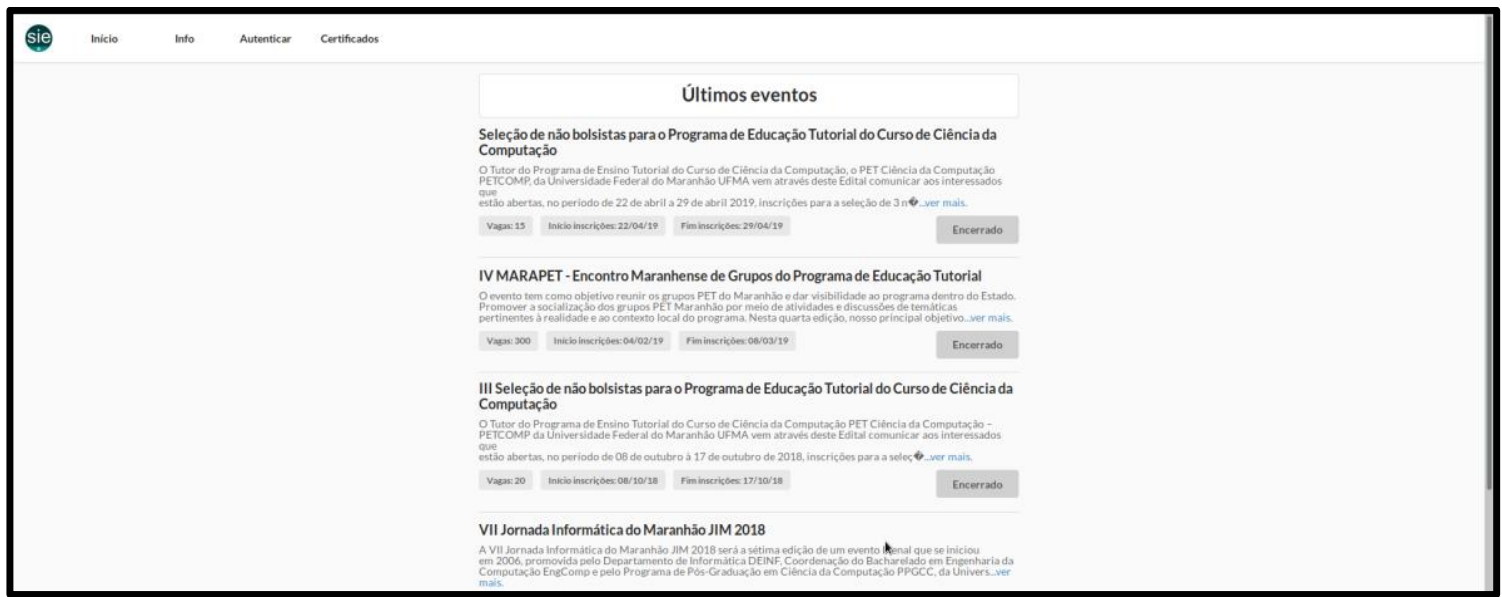

Figura 2. SiePET - Sistema desenvolvido para controle de eventos 


\subsection{Grupo de Acompanhamento Discente}

Segundo Pascoal et. al [2015], os principais fatores determinantes para a evasão no curso de computação são: domínio de interpretação e compreensão de texto, habilidade de resolução de problemas, embasamento matemático e experiência prévia em programação, computadores e softwares. De acordo com Hinterholz [2009], outros problemas são: dificuldade em permutar entre assimilar o conteúdo e desenvolver o raciocínio lógico, falta de motivação devido à crença de que a aprendizagem sobre o assunto é um obstáculo e despreparo. Pensando nesse contexto, o PET Ciência da Computação criou o Grupo de Acompanhamento de Discente (GAD). Ele é um serviço de apoio pedagógico [Scarparo et al. 2008] que visa oportunizar o desenvolvimento de habilidades técnicas e aprofundamento teórico, proporcionando aperfeiçoamento acadêmico. Além disso, o GAD visa contribuir para a formação integrada do aluno em atividades de ensino, facilitando o processo de ensino-aprendizagem de discentes, assim como a criação e adaptação de novas metodologias.

O GAD articula a criação de um grupo de estudo de disciplinas de cálculo e programação, que utilizam ferramentas online interativas, como Wanda e Cosmo (desenvolvidas por alunos de graduação e pós-graduação da própria UFMA), ocorrendo o acompanhamento à distância e presencial. $\mathrm{O}$ acompanhamento presencial e facultativo é realizado semanalmente. As atividades são conduzidas para as disciplinas de Algoritmos, Linguagem de Programação e Estrutura de Dados, onde são aplicados os planejamentos propostos pelos monitores, havendo momentos para realizar exercícios e esclarecer as dúvidas. Também é realizado o acompanhamento à distância, que vai além do laboratório, através das ferramentas Slack ${ }^{2}$ e Google Classroom ${ }^{3}$, dando assistência quando há dúvidas, e estímulos através de atividades e desafios extras.

Uma das melhorias que se busca implementar nesta atividade é a inserção de vídeo-aulas com conteúdos específicos a determinados tópicos, a fim de demonstrar e comparar diferentes abordagens e sua performance, frente a um determinado problema.

\subsection{Vem com a Computação}

Esta ação visa ensinar aspectos de aplicação de computação para a comunidade local, considerando as dificuldades enfrentadas pela população quanto à falta de inclusão digital. Entre as problemáticas abordadas, temos: utilização de ferramentas computacionais corriqueiras por parte de idosos, como redes sociais; dificuldades profissionalizantes do ponto de vista dos jovens da periferia; inclusão do público feminino em cursos da área de ciências exatas, particularmente, computação. Em todos os cenários listados acima, existem indivíduos que desejam usar tecnologias de computação, entretanto, os mesmos têm acesso restrito à elas.

A metodologia para realização desta ação seguiu duas etapas realizadas em paralelo: na primeira, foram apresentadas palestras e minicursos na área de computação, onde o público foi formado por mulheres no ensino médio de escolas públicas; na segunda, foi apoiado o público da terceira idade e a comunidade em geral através da realização de cursos e palestras.

\footnotetext{
${ }^{2}$ https://slack.com/

${ }^{3}$ https://classroom.google.com
} 
Em aulas direcionadas aos idosos, foi ensinado como utilizar as funcionalidades básicas de um smartphone e aplicativos específicos. Em outras atividades houveram palestras motivacionais para seguir um curso na área de computação. Com esta atividade, os petianos podem desenvolver aspectos sociais e incentivar a colaboração entre o grupo, através de ações de extensão. A Figura 3 apresenta uma das palestras realizadas para alunas em uma escola, com o objetivo de apresentar conceitos de computação para meninas do ensino médio.

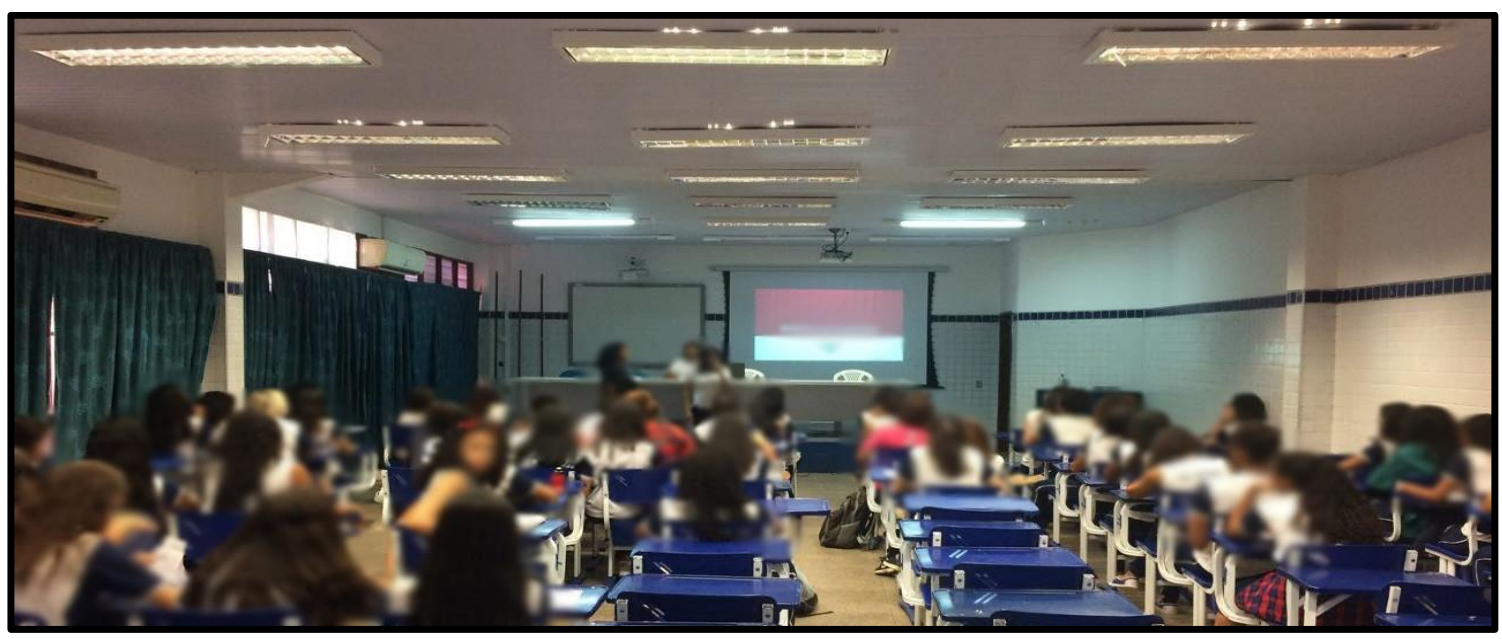

Figura 3. Palestra para meninas do ensino médio de uma escola pública

\subsection{Pesquisa Aplicada}

Os docentes e discentes do curso de Ciência da Computação da UFMA realizam várias atividades de pesquisa, ensino e extensão, aplicando seus conhecimentos em áreas como: computação móvel, visão computacional, jogos educacionais, engenharia de software, computação gráfica, robótica, desenvolvimento de aplicativos, entre outros. O objetivo desta ação é inserir o grupo PET Ciência da Computação em atividades de pesquisa científica, proporcionando o contato e aproximação de áreas de interesse individual de cada petiano. A ação contribui para desenvolver uma formação crítica e colaborativa, além de desenvolver as habilidades de escrita, defesa e apresentação oral de trabalhos, possibilitando ainda a realização de iniciações científicas e tecnológicas, como também incentivar o discente a considerar a realização de uma pós-graduação.

A prática é realizada através da cooperação com os laboratórios de pesquisa, núcleos de pesquisa e articulação com os demais docentes sobre a inserção de alunos do PET em atividades correlatas à pesquisa. Uma vez envolvido em uma destas atividades, o aluno utiliza parte da sua carga horária para desenvolver a pesquisa, escrever artigos científicos ou relatórios, e preparar apresentações dos conteúdos aprendidos. O tutor pode atuar como revisor dos trabalhos elaborados, junto aos docentes específicos de cada laboratório ou área de pesquisa. Os alunos também são encorajados a submeter os trabalhos preparados, em eventos científicos para publicação e divulgação.

Para aumentar o escopo desta atividade, espera-se que o petiano possa aplicar e acompanhar a aplicação de sua pesquisa, como forma de registrar seu desenvolvimento e permitir o amadurecimento da mesma durante a graduação até a pós-graduação. 


\subsection{Grupo de Saberes}

O grupo de saberes é uma atividade que promove um ciclo contínuo de palestras, minicursos e debates. As atividades são oferecidas para os petianos de computação e ao público geral como resultados das atividades de Pesquisa Aplicada, Fábrica de Software e GAD. As palestras, seminários e minicursos têm temática motivacional, tecnológica ou instrutiva. O objetivo da temática motivacional é abordar temas que relacionem e posicionem o discente de Ciência da Computação no mercado, no meio social e político. A temática tecnológica aborda a utilização e o desenvolvimento de tecnologias e ferramentas de ponta. A temática instrutiva busca demonstrar metodologias e melhorias aplicadas durante as atividades de ensino.

O propósito é que inicialmente se crie uma massa crítica que posteriormente possa ser replicada por outros discentes do curso de Ciência da Computação e atores atendidos diretamente com a ação. Além disso, espera-se prover formação tecnológica de ponta e assim qualificar os alunos para as necessidades futuras, trazer melhorias para o curso, para a educação e para a sociedade, meios para a socialização dos resultados, publicações e ainda construir conhecimentos complementares à grade curricular do curso, moldando um espírito empreendedor.

Para melhorar esta atividade, busca-se criar um banco de dados para registrar trabalhos, pesquisas, autores, área de pesquisa, a fim de facilitar o contato e parcerias entre novos petianos, autores e interessados pelo assunto.

\subsection{Evento Acadêmico de Computação}

O Evento Acadêmico de Computação é realizado de forma anual organizado pelo grupo PET Ciência da Computação em resposta aos anseios da comunidade discente de computação que carecia de um momento dentro da UFMA para a divulgação dos trabalhos acadêmicos, pesquisas e softwares desenvolvidos. O evento funciona como um espaço amplo de divulgação de trabalhos acadêmicos, onde docentes e discentes se revezam, socializando e trocando experiências sobre os grupos de pesquisa, tecnologias, contribuições científicas, dentre outras. A atividade consiste numa iniciativa de extensão ao contribuir com a difusão científica e tecnológica à comunidade da área em geral. Também incentiva práticas de ensino sobre gerenciamento e controle de atividades para os petianos, assim como incentiva a pesquisa, criatividade e espírito crítico dos mesmos ao buscar soluções para resolver eventuais problemas durante a realização do evento.

O evento também tem por objetivo apresentar o curso para os novos alunos e divulgar os trabalhos desenvolvidos no curso de Ciência da Computação da UFMA e de outras instituições. Durante o evento são realizadas competições de aplicativos, jogos e programação, além de possibilitar a troca de experiências dos estudantes. Finalmente, o evento promove o diálogo com profissionais de diferentes áreas que englobam a computação, expondo assim os desafios e possibilidades de atuação da mesma.

O EAComp na sua VI Edição. O evento é planejado através de reuniões com o grupo PET Ciência da Computação. Na primeira reunião, um grupo de petianos é eleito como coordenadores do evento. Esses coordenadores indicam membros para auxiliá-los diretamente. $\mathrm{O}$ tutor atua como facilitador entre as várias entidades e atores da universidade. $\mathrm{O}$ grupo então define a programação baseada em minicursos, palestras, competições, apresentação de trabalhos e laboratórios. A programação é baseada na tendência atual da área e tende a atender os anseios do público diretamente envolvido no curso de Ciência da Computação. Por fim, o evento é divulgado em todos os cursos da 
área de computação, em instituições públicas e particulares, assim como em instituições que possuam cursos técnicos na área. Durante o evento, a comissão formada pelos discentes atua para a realização integral do evento, sendo que o papel do tutor consiste em orientar, ajudar e facilitar os processos. Ao final do evento, é disponibilizado um questionário objetivo de avaliação aos participantes através de um sistema de gerenciamento de eventos, desenvolvido pelos discentes do grupo PET Ciência da Computação, a fim de coletar sugestões positivas e negativas que permitam alcançar melhorias no evento, com o intuito de torná-lo cada vez mais relevante e produtivo não apenas dentro da área da computação, mas para o público em geral.

\section{Resultados}

As atividades do grupo PET Ciência da Computação têm desempenhado um papel importante no desenvolvimento pessoal, profissional e acadêmico dos futuros cientistas da computação, mais especificamente dos petianos envolvidos no programa. Para avaliar os resultados das atividades propostas pelo grupo PET Ciência da Computação, foram realizadas entrevistas e/ou aplicados questionários com os participantes das diversas ações realizadas. A preparação das questões seguiu meios informais, com o objetivo de coletar a percepção dos participantes de modo a relatar: aspectos positivos, problemas nas ações realizadas e sugestões de melhoria para uma nova reaplicação dessas ações. A seguir são apresentados alguns dos pontos levantados pelos participantes.

Com relação à realização da ação Fábrica de Software, 6 alunos que participaram do projeto foram entrevistados. Todos os alunos indicaram que o projeto aumenta sua valorização profissional devido aos conhecimentos adquiridos referentes à criação de sites e tecnologias específicas, como HTML, CSS, Javascript, PHP; conceitos sobre modelagem e implementação de banco de dados e aplicação de metodologias ágeis. Os alunos indicaram que o projeto é extremamente importante, visto que ele permite: o contato com tecnologias atuais, obter qualificação profissional e compreender quais são os principais problemas que ocorrem durante o desenvolvimento de um software, em áreas de gerenciamento de projetos, aplicação de tecnologias de desenvolvimento e engenharia de requisitos. Adicionalmente, os alunos participantes desta ação indicaram que graças à sua participação, eles conseguiram posteriores estágios em empresas ou em projetos de desenvolvimento tecnológico.

Para a ação GAD, um total de 39 discentes (entre monitores e participantes) responderam a um questionário relatando sua percepção sobre a ação. Os alunos indicaram que foram aprovados nas disciplinas em que receberam monitoria e que o índice de aprovação dos alunos que não frequentaram as aulas do projeto foi menor. Além disso, $90 \%$ dos alunos que frequentaram a monitoria indicaram que a mesma teve um impacto positivo no aprendizado dos conteúdos das disciplinas, além de permitir a integração com os discentes do curso. Entre os problemas citados pelos alunos, 50\% afirmaram que tiveram dificuldades em comparecer às aulas devido aos horários incompatíveis. Além disso, os discentes indicaram que é necessária uma melhor comunicação entre o professor e os monitores da disciplina, principalmente quando se diz respeito aos procedimentos metodológicos de orientação, conceitos apresentados e material preparado.

Quanto às ações envolvendo a realização de pesquisa aplicada, treinamentos e cursos (Pesquisa Aplicada, Vem com a Computação e Grupo de Saberes, 
respectivamente), os alunos indicaram que estas ações permitiram abrir portas no mercado de trabalho e/ou especialização em um grupo de pesquisa, devido ao incentivo e aprendizado de novos conceitos e tecnologias de desenvolvimento de software ou à maneira de resolver problemas de forma sistemática. Verifica-se ainda o incentivo à continuação na carreira acadêmica, através de pesquisa em parcerias com laboratórios e até mesmo a inserção em um laboratório de pesquisa ou participação em projetos de pesquisa/tecnológicos ao sair do programa. Percebe-se ainda a participação em projetos que atingem a comunidade através do trabalho com idosos e meninas do ensino médio, com o desenvolvimento de objetos tecnológicos que tenham como público alvo a própria comunidade (acadêmica ou local), assim como desenvolvimento e publicação de trabalhos científicos.

O Evento Acadêmico de Computação da UFMA tornou-se um espaço regular de encontro de discentes e docentes do bacharelado, para o qual os discentes se preparam para apresentar trabalhos acadêmicos. Os trabalhos vão desde os resultados das pesquisas que ocorrem nos laboratórios até aplicativos desenvolvidos individualmente ou em grupo. De acordo com as informações colhidas de participantes das edições anteriores do evento, houve uma grande melhoria para o curso, para a educação e para a sociedade, sendo um meio para a socialização dos resultados e publicações. Além disso, o grupo PET Ciência da Computação tem contribuído para tornar o ambiente acadêmico mais dinâmico, divulgar o curso e estimular o desenvolvimento de trabalhos autônomos por parte dos discentes, alcançando assim o objetivo central do evento.

Com relação aos trabalhos publicados de outros grupos PET de computação, o trabalho de Lucena et al. [2018] apresenta os resultados de 7 ações, como: monitorias, publicação de revista, reaproveitamento de lixo tecnológico, apoio ao ensino de ferramentas nas escolas, semana acadêmica de ciência da computação, treinamentos da OBI e maratona de programação. Embora as atividades sejam similares, o grupo foca mais em ensino e extensão. Além disso, o grupo PET Ciência da Computação pode incorporar atividades relatadas no trabalho citado, como reaproveitamento de lixo eletrônico, assim como a possibilidade de participar de atividades acadêmicas específicas da computação, como a maratona de programação. Finalmente, quanto às oportunidades de melhoria, o artigo de Lucena et al. [2018] foca mais na metodologia aplicada do que no que poderia ser aplicado para mitigar os problemas que podem surgir na eventual reaplicação das atividades. Nesse contexto, este trabalho pode sugerir aspectos a serem analisados ou repensados por grupos PET interessados em adotar as ações sugeridas.

Finalmente, o trabalho de Ferreira et al. [2015] foca mais no aprendizado de tecnologias através do desenvolvimento de sistemas computacionais ou aplicação de conhecimentos em áreas como robótica. Os autores apresentam uma discussão quanto à metodologia adotada e os resultados encontrados, porém, sem entrar em detalhes sobre como a metodologia geral seria implementada.

\section{Conclusões}

Este artigo apresentou um conjunto de ações que foi realizado pelo grupo PET Ciência da Computação da Universidade Federal do Maranhão, visando o envolvimento dos discentes no curso de computação. Os resultados apontam para o sucesso das atividades com oportunidades de melhoria ao disseminar cursos, participar no desenvolvimento de software, realizar pesquisas e participar da organização de um evento na área de 
computação. Quanto à atividade de divulgação, como Vem com a Computação e Grupo Saberes, espera-se ampliar o público alvo, além da criação de um banco de informações de autores para facilitar o contato de interessados para possíveis parcerias. $\mathrm{Na}$ atividade de desenvolvimento, espera-se melhor cooperação do público alvo quanto às informações e parcerias com o grupo PET Ciência da Computação, como uma forma de alimentar sites e aplicativos com informações relevantes pertinentes à comunidade, assim como a participação de antigos petianos através de minicursos e consultorias, de modo a facilitar a entrada de novos petianos no desenvolvimento, e também dar direcionamento frente aos problemas complexos. A atividade de pesquisa, embora nem sempre o petiano conclua a pesquisa durante seu período no PET, o mesmo continua seu desenvolvimento após sua saída, o que permite mais engajamento por parte do discente em atividades extracurriculares. Espera-se ainda que por meio das pesquisas o petiano possa avançar a mesma para padrões de Trabalho de Conclusão de Curso (TCC). Quanto à atividade de organização de eventos, espera-se que o petiano possa quantificar os pontos positivos e negativos do evento, assim como a aplicação e efetividades de possível melhorias, além de aplicação da relevância do evento para diferentes públicos, principalmente os não relacionados à computação. Finalmente, quanto às atividades relatadas pelos outros grupos PET da área de computação, pretende-se incorporar às ações e/ou procurar parcerias para mais envolvimento da comunidade acadêmica na participação de eventos nacionais de computação, como maratonas e competições. Espera-se que com a divulgação dos resultados apresentados neste artigo, outros grupos PET sejam motivados a participar das atividades relatadas, e/ou sejam construídas parcerias, de modo a incentivar a capacitação dos alunos de Ciência da Computação no que diz respeito ao ensino, pesquisa e extensão.

\section{Referências}

de Deus Júnior, G. A., \& Chaveiro, E. F. (2014). Programa de Educação Tutorial na Escola de Engenharia Elétrica, Mecânica e de Computação (EMC) da UFG: As Dimensões do Trabalho e da Formação. Revista Eletrônica Engenharia Viva, 1, 55-73.

Ferreira J., Ribeiro, J., Freitas, A., Barros, G., Santos, I., Santos, H., Chaves, M., Albuquerque, A., Silva, B., Rezende, L., Bezerra, V., Moraes, A., Oliveira, A. (2015) Educação Tutorial em Ciência da Computação: uma proposta de sistematização $23^{\circ}$ WEI - Workshop sobre Educação em Computação.

Hinterholz, O. (2009). Tepequém: uma nova Ferramenta para o Ensino de Algoritmos nos Cursos Superiores em Computação. In XVII-Anais do Workshop sobre Educação em Informática (Vol. 20, p. 21).

de Lucena, L. B., de Andrade, G. L. C., Soares, E. D. F. A., de Oliveira, Á. G. G., Batista, L. M. D. S. D., Gomes, D. A., \& de Lima, R. W. (2018). As Ações do Pet no Desenvolvimento do Curso de Ciência da Computação. In $26^{\circ}$ Workshop sobre Educação em Computação (WEI 2018), SBC.

Martins, I. L. (2007). Educação Tutorial no ensino presencial: uma análise sobre o PET. PET-Programa de Educação Tutorial: estratégia para o desenvolvimento da graduação. Brasília: Ministério da Educação.

Moreno, V., Cavazotte, F., \& de Farias, E. (2009). Novos Desafios para o Profissional de TI: Estudo de Caso de uma Empresa de Prestação de Serviços de Tecnologia da Informação. Revista de Gestão da Tecnologia e Sistemas de Informação, 6, 437-462. 
Pascoal, T. A., de Brito, D. M., \& do Rêgo, T. G. (2015). Uma abordagem para a previsão de desempenho de alunos de Computação em disciplinas de programação. Nuevas Ideas en Informática Educativa TISE, 2015, 454-458.

Scarparo Haag, G., Kolling, V., Silva, E., Bastos Melo, S. C., and Pinheiro, M.(2008). Contribuições da monitoria no processo ensino-aprendizagem em enfermagem. Revista Brasileira de Enfermagem, 61(2). 